\title{
Hydrocarbon exposure, pancreatitis, and bile acids
}

\author{
P Hotz, J Pilliod, R Bourgeois, M-A Boillat
}

\begin{abstract}
The data on hydrocarbon induced pancreatitis are conflicting. This question was therefore studied in a non-selected population exposed to hydrocarbons and in "formerly" exposed workers. Neither the past clinical history nor the pancreatic tests provided any evidence for a causal relation between exposure and pancreatitis. No signs of hydrocarbon induced liver damage were seen either. As a healthy worker effect cannot be totally excluded, however, a case-control study in a group of patients suffering from non-alcohol induced pancreatitis could give useful indications for finally excluding the possibility of pancreatitis being induced by hydrocarbons.
\end{abstract}

A floor layer was recently referred to us because of an apparently temporal relation between gluing in confined spaces and attacks of pancreatitis. A few case reports exist that suggest a link between exposure to hydrocarbons or organic solvents and pancreatitis. ${ }^{1-3}$ The study by Braganza et $a^{4}$ also presents some arguments for this hypothesis but it did not include an exposure score or a control group. On the other hand, the findings of Orbaeck et al ${ }^{5}$ do not support the hypothesis but their study group was highly selected and the study did not specifically consider the question of occupational factors as a cause of pancreatitis. In a recent review on chemicals toxic to the pancreas the toxicity of dimethylbenz(A) anthracene in rats is mentioned but no comment is made on hydrocarbons or organic solvents. ${ }^{6}$ Finally, Pedersen $e t a l^{7}$ noted a decreased amylase activity during exposure of human volunteers to white spirit.

These conflicting reports prompted us to study this question in a population of floor layers. People with exposure to motor exhaust fumes or to miscellaneous organic solvents were included as well to provide populations similar to those of Braganza et $a l^{4}$ and Orbaeck et $a l .^{5}$ An induction measure was included as Braganza et al $^{4}$ suggested a link between enzyme inducers and pancreatitis. We also measured

Institute of Occupational Health, Bugnon 19, CH1005 Lausanne

P Hotz, J Pilliod, R Bourgeois, M-A Boillat the concentration of bile acid to see whether this test is more sensitive than measurement of alanine aminotransferase or $\gamma$-glutamyltransferase activity for detecting liver damage induced by hydrocarbons.

\section{Subjects and methods}

As the demonstration of a dose effect relation would be a good argument for ascribing a role for exposure to hydrocarbons in the genesis of pancreatitis, we made four exposure categories (no, low, moderate, and heavy exposure) according to a questionnaire that has been described in detail elsewhere. ${ }^{8}$ The study population comprised two groups.

Group A consisted of 22 controls (no exposure), 40 roadmen (slight exposure), 34 printers (moderate exposure), 106 floor layers (heavy exposure), six workers exposed to miscellaneous hydrocarbons (slight, moderate, or heavy exposure), 21 "formerly" exposed workers, who had had previous exposures but were no longer exposed to hydrocarbons, and one ex-printer who changed his job but was still exposed in the moderate exposure category. The median time elapsed since last exposure for the "formerly" exposed workers was $4 \cdot 2$ years (range $0 \cdot 2-27 \cdot 9$ years). All the subjects were men and were working at the time of the examination. No patient referred to us for investigation of an occupational disease was eligible, thus avoiding a recall bias. The eligibility criteria were the same as previously described. ${ }^{8}$ No workers refused to cooperate, but some analyses were lost because of organisational or technical problems.

The floor layers came from 20 plants and the number of workers in each plant varied from two to 18 . We made every effort to sample different working conditions but were unable to verify the representative nature of our sample because no occupational register existed that would allow it. A preliminary field survey showed that glues based on organic solvents were used most of the time, often in confined spaces, and without any protection device. The organic solvent vapours were a complex mixture that contained at least $\mathbf{4 0}$ different hydrocarbons. The main air contaminants appeared to be toluene or methanol. This and the fact that the vapour concentrations varied greatly and rapidly led us to consider that biological monitoring was not achievable.'

All printers came from the same plant, where they were exposed to toluene. Their exposure has been described elsewhere. ${ }^{10}$ 
The roadmen are responsible for the maintenance of the roads and of their surroundings. They are exposed to exhaust fumes outdoors and irregularly use pesticides, paints, or tar products. Complaints about at least one acute hydrocarbon induced work related symptom (defined as suggested by a recent WHO report ${ }^{11}$ as feeling drunk, or having an unspecific gastric disturbance, dizziness, nausea, alcohol intolerance, or drowsiness) were not reported by this group. In this respect they differed from floor layers and printers. Owing to their occupational history, their lack of hydrocarbon induced work related symptoms, and the field survey of Darby $e t$ $a l,{ }^{12}$ we considered that their classification into the slight exposure category was appropriate.

Group $B(n=14)$ consisted of patients referred to us in 1989 for investigation of possible occupational problems and whose clinical history disclosed an occupational exposure to hydrocarbons. They were included in a second step of the analysis as a heterogeneous group for present job and exposure score.

Besides the present job, four exposure estimates were used: exposure duration, exposure score, reporting of symptoms induced by hydrocarbons, and complaints about work related disturbances of the digestive system. Exposure duration (ED; years) and exposure score (ES; arbitrary units, years $\times$ exposure intensity factor) were assessed on the basis of 27 questions as described previously. ${ }^{8}$ For analysis of variance the group was split according to the median exposure score or to a 10 year limit for comparing our results with those of Orbaeck et al. ${ }^{5}$ As present work related symptoms could be a better indicator of the health effects of hydrocarbons a subgroup of workers complaining of at least one work related acute symptom (as defined above) and a subgroup of workers reporting at least one work related digestive symptom were created. The digestive symptoms (DS) were defined as any trouble affecting appetite or thirst, alcohol or food intolerance, gastric disturbance, vomiting, abdominal pain, or intestinal peristalsis provided that they had an obviously work related pattern.

Clinical and occupational history were recorded and samples of blood and urine were collected exactly as described previously. ${ }^{8}$ Alcohol consumption was defined according to the French translation of the questionnaire described by Rollason et al. ${ }^{13}$ For some analyses of variance the non-drinkers and those that only drank socially (not daily) were separated from people drinking alcohol daily.

Obesity was estimated by the body mass index (BMI)—namely, body weight $(\mathrm{kg}) /$ height $(\mathrm{m})^{2}$.

A disease of the digestive system (DS) was defined as any history of disease affecting the alimentary or the biliary tract, the liver, or the pancreas. As a regular drug intake in the previous month can influence many tests, this factor was considered as well and workers with a DS disease or a regular drug intake, or both, were pooled together as the "ill" subgroup.

Isoamylases were measured with the Phadebas isoamylase test (Pharmacia Diagnostics). The pancreatic isoamylase:creatinine clearance ratio (ICCR) was calculated as

$$
\begin{gathered}
\frac{\text { urinary pancreatic isoamylase activity }}{\text { serum pancreatic isoamylase activity }} \times \\
\frac{\text { serum creatinine }}{\text { urinary creatinine }} \times 100
\end{gathered}
$$

Creatinine and lipase and $\gamma$-glutamyl transferase (GGT) activity were measured with standard methods (Boehringer Diagnostica); also alanine aminopeptidase activity (ALAT) (Roche Diagnostica) and bile acid concentration (Merck Diagnostica). Apart from the ICCR, all values are expressed as per cent of the upper limit of the reference range to make comparisons easier. The reference range for the ICCR is $2 \cdot 64+0.86 \%(n=26) .{ }^{14}$ All analyses were done in duplicate according to the usual rules of quality control.

All statistics were performed with the statistical package for social sciences. ${ }^{15}$ If necessary data were transformed to logarithms. The relative role of the different factors and variables was studied by multiple analysis of variance or by backward multiple regression analysis. Differences in prevalence were evaluated by the Pearson $\chi^{2}$ test.

\section{Results}

The results for group A are presented first. Table 1 summarises the main clinical and laboratory data.

People with an ES above the median reported work related acute symptoms more often than those with an ES below the median ( $p=0.01, \chi^{2}$ test) but not DS symptoms $(p=0 \cdot 28)$, in their clinical history. Differences between workers were more evident if the present job was considered (table 2).

A clinical history of disease of the exocrine pancreas was found in three workers who all belonged to the exposed group. An occupational origin could, however, be excluded as trauma was the cause in one case and mumps in another. In the third case the only attack of pancreatitis occurred before any exposure to hydrocarbons. Only one case of work related abdominal pain was found (in the lower exposure score group).

As early pancreatic disturbance can be difficult to diagnose some cases could have been misclassified as having a disease affecting the digestive system. Therefore, the analysis was repeated with this broader diagnostic category. As regular alcohol consumption (particularly the highest drinking cate- 
Table 1 Clinical and laboratory data for group $A$

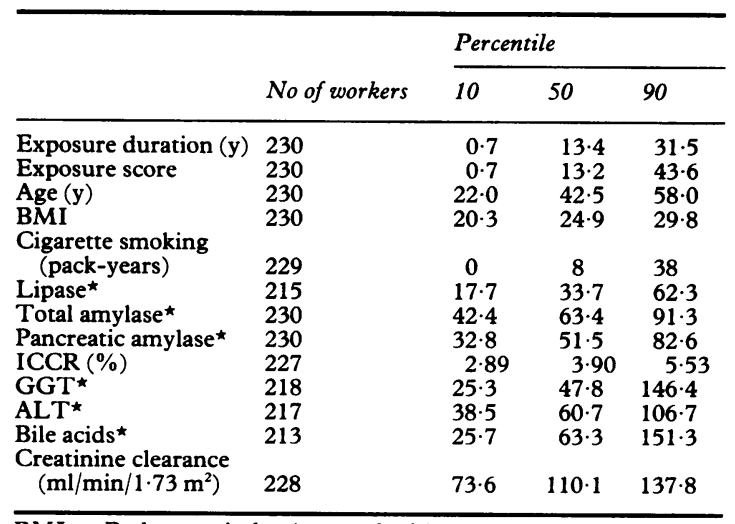

BMI = Body mass index (see methods)

* Per cent of the upper limit of the reference range.

ICCR = Pancreatic isoamylase: creatinine ratio.

gories) was more often reported in the subgroups with an ES above the median or an ED equal to or greater than 10 years $(p=0.002)$ the slightly increased prevalence of diseases affecting the DS in these subgroups $(p=0.02)$ could hardly be explained only by the exposure; this difference in prevalence did not reach statistical significance after excluding workers in the heaviest drinking class (regularly six or more drinks daily).

Present job was not associated with alcohol consumption or with DS diseases. The prevalence of DS diseases was not greater in the "formerly" exposed compared with the presently exposed workers or with the controls. The floor layers did not report a history of DS disease more often than other workers $(p=0.73)$ although they complained more often about work related digestive symptoms at their clinical history.

A three way factor analysis including the factors ES (or ED), alcohol consumption, and "ill" group disclosed no exposure effect on the activities of total amylase, of pancreatic isoamylase, or on the ICCR, and only a weak relation between ES or ED and lipase activity. This variance analysis was repeated with the factors present job, present work related acute, or DS symptoms as exposure markers instead of ES or ED groups. This did not disclose any meaningful relation between exposure to hydrocarbons and pancreatic tests either. There was no significant interaction between the exposure surrogates and the factors alcohol consumption or "ill" subgroup.

These results were further tested in a multiple regression analysis with the individual ES or ED. As age, ${ }^{16}$ cigarette smoking, ${ }^{17}$ and body weight ${ }^{18}$ are possible confounding factors, they were also included. The main result was that the effect of exposure on lipase activity disappeared after taking these variables into account.

Alcohol consumption, BMI, and regularly taking drugs all increased the activity of the inducible enzyme GGT in a statistically significant way but the exposure estimates (exposure duration, exposure score, work related acute or DS symptoms) had no effect. The interactions between ES or ED and alcohol consumption played no part either. Activity of ALAT and concentration of bile acid were not related to these exposure estimates. Table 3 summarises the main results.

Body weight and BMI were not associated with the reporting of acute or DS symptoms or with the exposure score ( $p>0.05$ in all cases).

In group B two patients had a history of pancreatic disturbance. The first one, a floor layer, was a heavy drinker who needed a stay in hospital and treatment with disulfiram because of his alcohol consumption. He gave up drinking and one year after resuming his work he had had no pancreatic problem. The second patient had suffered from several attacks of pancreatitis but they began at least two years before any occupational exposure to hydrocarbons. His consumption of alcohol was in the highest category according to the scale used. Including the test results of group B in the multiple regression analysis did not modify the results.

Table 2 Present job and number of workers reporting at least one obviously work related acute or digestive system symptom

\begin{tabular}{|c|c|c|c|c|c|c|}
\hline \multirow[b]{2}{*}{ Job category } & \multirow{2}{*}{$\begin{array}{l}\text { Exposure score } \\
\text { median }(P 90)\end{array}$} & \multicolumn{2}{|c|}{ Acute symptom (s) (No(\%)) } & \multicolumn{3}{|c|}{ Digestive symptom(s) $(\text { No }(\%))^{\star \star}$} \\
\hline & & Yes & No & Yes & No & \\
\hline $\begin{array}{l}\text { Controls } \\
\text { Roadmen } \\
\text { Printers } \\
\text { Floor layers } \\
\text { "Formerly" exposed workers } \\
\text { Miscellaneous exposures }\end{array}$ & $\begin{array}{rr}0 & (0) \\
13 & (18) \\
19 & (36) \\
21 & (53) \\
10 & (39) \\
4 & (9)\end{array}$ & $\begin{array}{rr}0 & (0) \\
0 & (0) \\
5 & (26) \\
63 & (64) \\
0 & (0) \\
0 & (0)\end{array}$ & $\begin{array}{rr}18 & (100) \\
30 & (100) \\
14 & (74) \\
35 & (36) \\
19 & (100) \\
4 & (100)\end{array}$ & $\begin{array}{rr}0 & (0) \\
0 & (0) \\
1 & (5) \\
18 & (22) \\
0 & (0) \\
0 & (0)\end{array}$ & $\begin{array}{r}16 \\
28 \\
18 \\
63 \\
17 \\
4\end{array}$ & $\begin{array}{r}(100) \\
(100) \\
(95) \\
(78) \\
(100) \\
(100)\end{array}$ \\
\hline
\end{tabular}

${ }^{\star} \mathrm{p}<0.0001 ;{ }^{\star \star} \mathrm{p}<0.003$ (Pearson $\chi^{2}$ test).

P90 $=90$ th percentile.

Workers with the same symptoms that were of non-occupational origin are excluded from the data. 
Table 3 Multiple regression analysis

\begin{tabular}{|c|c|c|c|c|c|c|}
\hline \multirow[b]{2}{*}{ Dependent variable } & \multicolumn{5}{|c|}{ Partial regression coefficients for } & \\
\hline & Age & $B M I$ & Body weight & $\begin{array}{l}\text { Alcohol } \\
\text { consumption }\end{array}$ & \multicolumn{2}{|l|}{ Drug intake } \\
\hline Lipase & $0.007^{\star \star \star}$ & NS & $-0.58^{\star}$ & NS & NS & $0 \cdot 11$ \\
\hline Pancreatic amylase & $0.004^{\star \star \star}$ & NS & $-0.60 \star \star$ & NS & NS & 0.08 \\
\hline ICCR & NS & NS & NS & $0.03^{\star \star}$ & NS & 0.04 \\
\hline GGT & NS & $0.03 \star \star \star$ & NS & $0 \cdot 11^{\star \star \star}$ & $0 \cdot 16^{\star \star \star}$ & 0.36 \\
\hline ALAT & NS & $0.02^{\star \star \star}$ & NS & $0.04^{\star \star}$ & NS & $0 \cdot 11$ \\
\hline Bile acids & NS & NS & $-0.85^{\star \star}$ & $0.04^{\star}$ & NS & 0.05 \\
\hline
\end{tabular}

${ }^{\star} \mathrm{p} \leqslant 0.05 ;{ }^{\star \star} \mathrm{p} \leqslant 0.01 ;{ }^{\star \star \star} \mathrm{p} \leqslant 0.001$.

NS $=$ Not significant.

The variables exposure score, exposure duration (y), cigarette smoking (pack-years), and the interaction between exposure and alcohol consumption did not show any consistent effect on the dependent variables.

\section{Discussion}

If hydrocarbons damage the pancreas either recurring acute attacks of pancreatitis with increased enzyme activity or a chronic pancreatitis with reduced enzyme activity would be expected. In both cases the ICCR should increase. Our field survey did not disclose such relations, however, between pancreatic tests and exposure to hydrocarbon. This accords with the findings of Orbaeck $e t a l^{5}$ and allows some generalisation of their conclusions as we did not exclude workers with less than 10 years exposure.

The diagnosis of pancreatic disease can be difficult as outlined by Ammann. ${ }^{19}$ The tests we used, however, seem to be adequate for a field survey. ${ }^{14} 1920$ Some others, such as the bentiromide function test, may be preferable but are too time consuming for use as screening methods. It is emphasised that the ICCR is increased in quiescent chronic pancreatitis as well ${ }^{14}$ and that an exocrine pancreatic dysfunction, which our tests aimed to detect, generally precedes endocrine dysfunction, particularly in chronic nonalcoholic pancreatitis. $^{21}$

Acute methanol intoxication can cause a toxic pancreatitis but although our floor layers could inhale, at least theoretically, high concentrations of methanol vapours as well as other organic solvents, their pancreatic test values were similar to those of less exposed workers.

The occupational history of our workers was similar to that of the subjects of Braganza et $\mathrm{al}^{4}$ so that differences in exposure cannot explain why we come to an opposite conclusion. A possible explanation is that the positive association described by Braganza et al $^{4}$ was due to chance; these authors had neither a control group nor a well defined assessment of exposure.

The rationale discussed by Braganza et $\mathrm{al}^{4}$ relies on a hypothetical hydrocarbon mediated enzyme induction. Our results have not provided support for this as GGT activity correlated neither with the exposure nor with an interaction between alcohol consumption and exposure. It could be argued that there are several tests of enzyme induction that do not always correlate with each other and that GGT activity was not an adequate test in the population we studied. Degree of GGT activity is thought to be a fairly good induction marker, however, ${ }^{22}$ and studies using excretion of D-glucaric acid or antipyrine clearance have presented a confusing picture ${ }^{1323-25}$ that does not clearly show a hydrocarbon stimulated induction of microsomal enzymes. That such an induction plays a part in the genesis of occupational pancreatitis seems, therefore, questionable.

Of the case reports mentioned earlier, the first ${ }^{1}$ deals with exposure to dimethylformamide but the clinical examination of three coworkers gave doubtful results and the conclusions should be viewed with caution. The second ${ }^{2}$ described an acute pentachlorophenol intoxication but the toxic pancreatitis could have been due to a systemic effect-namely the uncoupling of oxidative phosphorylation, and not to a direct toxic effect. In the third case report, Doesling et $a l^{3}$ did not fully exclude the possibility that the chronic pancreatitis they diagnosed in a painter exposed mainly to white spirit, was a random association.

The findings of Pedersen $e t a l^{7}$ are more difficult to explain as the exposure was well controlled; the volunteers were their own controls and a dose relation was found. Nevertheless, an effect of exposure was noted with only one of the three white spirits tested and the amylase activity decrease was less than $10 \%$. A possible explanation would be an analytical artifact. In any case, if the exposure was a cause of toxic acute pancreatitis, an increased (not a decreased) amylase activity would have been expected.

It has been emphasised recently that it is probably not very useful to screen a population exposed to hydrocarbons that do not have prominent hepatotoxic effects. ${ }^{25}$ This is supported by our results. More interesting is that we could not confirm that concentrations of bile acid are more sensitive markers of liver injury than activity of GGT or AAT as stated in a recent review. ${ }^{27}$ This discordance with the results of others could be explained by the fact that the 
influence of body weight was not taken into accoun$\mathrm{t}^{28-30}$ This influence could be related to differences in diet intake as described for amylase ${ }^{18}$ As body weight and exposure are not linked it cannot be argued that the variable body weight masked any effect of the exposure.

Clinical history did not disclose any relation between exposure to hydrocarbons or present job and pancreatitis even in the "formerly" exposed workers who could have left their job because of ill health ${ }^{31}$ or in the patients. A healthy worker effect is therefore not supported by our data but as our sample size is small a lack of power is possible and these observations should be confirmed in a case control study. Also a distinction between alcoholic and nonalcoholic chronic pancreatitis could be very useful as those two clinical entities seem not to have the same clinical history. ${ }^{21}$

In conclusion, we think that, apart from under some very unusual exposure conditions, there are no convincing arguments for considering an occupational exposure to hydrocarbons as a cause of toxic pancreatitis. These results should, however, be confirmed in a well designed case-control study.

We thank Professor J J Gonvers for his help with the measurement of bile acids and Mrs V Lietta for typing the manuscript.

1 Chary S. Dimethylformamide: A cause of acute pancreatitis? Lancet 1974;2:356.

2 Cooper RG, Macaulay MB. Pentachlorophenol pancreatitis. Lancet 1982;1:517.

3 Doessing M, Jacobsen O, Rasmussen SN. Chronic pancreatitis possibly caused by occupational exposure to organic solvents. Hum Toxicol 1985;4:237-40.

4 Braganza JM, Jolley JE, Lee WR. Occupational chemicals and pancreatitis: a link? Int J Pancreatol 1986;1:9-19.

5 Oerbaek P, Risberg J, Rosen I, et al. Effects of long-term exposure to solvents in the paint industry. Scand JWork Environ Health 1985;11:10-280.

6 Scarpelli DG. Toxicology of the pancreas. Toxicol Appl Pharmacol 1989;101:543-54.

7 Pedersen LM. Biological studies in human exposure to and poisoning with organic solvents: Kinetics, haemotology, and serum chemistry. Pharmacol Toxicol 1987;61:1-17.

8 Hotz P, Pilliod J, Soederstroem D, Rey F, Boillat MA, Savolainen $\mathrm{H}$. Relation between renal function tests and a retrospective organic solvent exposure score. $\mathrm{Br} \mathrm{J}$ Ind Med 1989;46:815-9.

9 Soederstroem D. Etude du systeme nerveux chez les poseurs de sols. Lausanne, Suisse: Universite de Lausanne, 1988:1-119. (Thesis.)

10 Boillat MA, Berode M, Droz PO. Examens preventifs dans l'heliogravure. Soz Praventivmed 1986;31:111-3.
11 World Health Organisation regional office for Europe/Nordic Council of Ministers. Chronic effects of organic solvents on the central nervous system and diagnostic criteria. Report. Joint meeting, June 1985, Copenhagen. Copenhagen/Oslo: WHO Regional Office for Europe/Nordic Council of Ministers, Environmental Health Series, 1985;5:1-273.

12 Darby FW, Willis AF, Winchester RV. Occupational health hazards from road construction and sealing work. Ann Occup Hyg 1986;30:445-54.

13 Hotz P, Guillemin M, Lob M. Study of some hepatic effects (induction and toxicity) caused by occupational exposure to styrene in the polyester industry. Scand J Work Environ Health 1980;6:206-15.

14 Hegarty JE, O'Donnell MD, McGeeney KF, Fitzgerald $O$. Pancreatic and salivary amylase/creatinine clearance ratios in normal subjects and in patients with chronic pancreatitis. Gut 1978;19:350-4.

15 SPSS'X. SPSS-XTM User's Guide. Chicago: SPSS Inc, 1988: 1-1072.

16 Bossuyt PJ, Van den Bogaert R, Scharpe SL, Van Maercke Y. Relation of age to isoenzyme pattern and total activity of amylase in serum. Clin Chem 1981;27:451-4.

17 Dubick MA, Conteas CN, Billy HT, Majumdar APN, Geokas MC. Raised serum concentrations of pancreatic enzymes in cigarette smokers. Gut 1987;28:330-5.

18 Kondo T, Hayakawa T, Shibata T, Sato Y, Toda Y. Serum levels of pancreatic enzymes in lean and obese subjects. Int $J$ Pancreatol 1988;3:241-8.

19 Ammann RW. Pankreasabklaerung. Ther Umsch 1987;44:444-9.

20 Moeller-Petersen J, Klaerke M, Dati F. Evaluation and comparison of cathodic trypsin-like immunoreactivity, pancreatic lipase and pancreatic isoamylase in the diagnosis of acute pancreatitis in 849 consecutive patients with acute abdominal pain. Clin Chim Acta 1986;157:151-65.

21 Ammann RW, Buehler H, Muench R, Freiburghaus AW, Siegenthaler $W$. Differences in the natural history of idiopathic (non-alcoholic) and alcoholic chronic pancreatitis. A comparative long-term study of 287 patients. Pancreas 1988; 2:368-77.

22 Goldberg DM. Structural, functional, and clinical aspects of gamma-glutamyltransferase. CRC Crit Rev Clin Lab Sci 1980;12:1-58.

23 Dossing M, Loft S, Sonne J, Schroeder E. Antipyrine and metronidazole metabolism during occupational exposure to gasoline. Int Arch Occup Environ Health 1988;60:115-8.

24 Skender LJ, Karacic V, Prpic-Majic D. Metabolic activity of antipyrine in workers occupationally exposed to trichloroethylene. Int Arch Occup Environ Health 1988;61:189-95.

25 Moretto A, Lotti M. Exposure to toluene increases the urinary excretion of D-glucaric acid. Br J Ind Med 1990;47:58-61.

26 Occupational Medicine Forum. Screening for hepatic damage. J Occup Med 1987;29:266-7.

27 Goldberg DM, Brown D. Advances in the application of biochemical tests to diseases of the liver and biliary tract: Their role in diagnosis, prognosis, and the elucidation of pathogenetic mechanisms. Clin Biochem 1987;20:127-48.

28 Franco G, Fonte R, Tempini G, CanduraF. Serum bile acid concentrations as a liver function test in workers occupationally exposed to organic solvents. Int Arch Occup Environ Health 1986;58:157-64.

29 Franco G, Santagostino G, Lorena M, Imbriani M. Conjugated serum bile acid concentrations in workers exposed to low doses of toluene and xylene. Br J Ind Med 1989;46:141-2.

30 Edling C, Tagesson C. Raised serum bile acid concentrations after occupational exposure to styrene: a possible sign of hepatotoxicity? Br J Ind Med 1984;41:257-9.

31 Miyake H, Harada H, Kunichika K, Ochi K, Kimura I. Clinical course and prognosis of chronic pancreatitis. Pancreas 1987; 2:378-85.

Accepted 11 June 1990 